\title{
How 3D Interaction Metaphors Affect User Experience in Collaborative Virtual Environment
}

\author{
Hamid Hrimech, ${ }^{1}$ Leila Alem, ${ }^{2}$ and Frederic Merienne ${ }^{1}$ \\ ${ }^{1}$ Arts et Metiers ParisTech, CNRS, Le2i Institut Image, 2 rue Thomas Dumorey, 71100 Chalon sur Saone, France \\ ${ }^{2}$ Networking Technologies, CSIRO ICT Centre, P.O. Box 76, Epping, NSW 1710, Australia
}

Correspondence should be addressed to Hamid Hrimech, hrimech@hotmail.fr

Received 12 March 2011; Revised 29 June 2011; Accepted 21 July 2011

Academic Editor: Kiyoshi Kiyokawa

Copyright (C) 2011 Hamid Hrimech et al. This is an open access article distributed under the Creative Commons Attribution License, which permits unrestricted use, distribution, and reproduction in any medium, provided the original work is properly cited.

In this paper we presents the results of our experimental study which aims to understand the impact of three interaction 3D metaphors (ray casting, GoGo, and virtual hand) on the user experience in a semi-immersive collaborative virtual environment (the Braccetto System). For each session, participants are grouped in twos to reconstruct a puzzle by an assemblage of cubes. The puzzle to reconstruct corresponds to a gradient of colors. We found that there is a significant difference in the user experience by changing the interaction metaphor on the copresence, awareness, involvement, collaborative effort, satisfaction usability, and preference. These findings provide a basis for designing 3D navigation techniques in a CVE.

\section{Introduction}

The CVEs technology aims at transforming the Internet networks into 3D navigable and populated spaces which allow the work of collaboration and the social play; however, the use of CVEs creates a mediatization of the collaboration; which means that we pass from a real situation face to face to a situation where we use a virtual world artificially created by computer programs to work together. Unfortunately, quite often this new way of working degrades among users some information necessary to the collaborative process, which includes the following.

(i) The actions of the partner.

(ii) The intentions of the partner.

(iii) The point of view of the partner.

The design of a CVE is regarded today as a real challenge. The problems are numerous and have a technological, cognitive, and social character strongly coupled.

In our study, we were focusing on the following research question "What is the impact of 3D interaction metaphors on the user experience in a CVE-oriented task." the understanding of these metaphors will help us to define the criteria, which are to be taken into account when designing a CVE. The next section reviews the literature, Section 2 presents our research approach. In Section 3 we describe the experiment and research hypotheses. Section 4 presents the experimental results which are discussed in Section as well as a conclusion and further work.

\section{Background and Previous Studies}

2.1. $3 D$ Interaction. In VR the manipulation of the objects is a very important element in $3 \mathrm{D}$ interaction. The quality of the VE depends on the level of interaction between the user and this environment. $\mathrm{n} 3 \mathrm{D}$ manipulation is a joint task between the real and the virtual world. In our everyday life we use our hands in order to interact with objects, the hand can be regarded as a perfect peripheral of interaction. It allows to manipulate different types of object in an effective and fast precise way. For example, if we want to carry a book from a point $\mathrm{A}$ to a point $\mathrm{B}$, we carry out the following stages:

one takes the book $\rightarrow$ one moves it $\rightarrow$ one over turns the book according to the desired orientation $\rightarrow$ one lays out the book.

According to Coquillart et al. [1], 3D interaction techniques are regarded as a set of methods or scenarios of the 
use of a hardware interface, allowing the user to carry out a specific task in a virtual environment (VE). According to Mine [2], 3D interactions are composed of four virtual behavioral primitives.

(i) Navigation: navigation in VE consists of two components: (1) a motor component of user movement or displacement in space and (2) a cognitive component or what one calls the "wayfinding," a cognitive process which makes it possible to define a way in a virtual environment by building a "cognitive map" of this space [3].

(ii) Selection: selection of an object among others is considered by [2] as the action of pointing an object and validating it.

(iii) Manipulation: manipulation indicates the modification of the state of an object often beforehand selected. This modification breaks up into two subtasks: the translation and rotation. Mine also include in the notion of manipulation, the modification of the object properties such as size, texture, or transparency [2]. But these actions are often carried out by using specific menus (widgets).

(iv) System control is a fundamental elementary task with any application enabling the dialogue between the user and the application. Indeed, the goal of the system control is the release of functions and options of the application.

In our work, we use a decomposition similar to that evoked previously, that is, for us the task of manipulation consists of the following.

(i) Selection: the selection consists of distinguishing an object among others (to take the book), the selection can then be presented in the following way:

$$
\text { Selection }=\text { pointing }+ \text { validation } \text {. }
$$

(ii) Translation: it is the task of change of the position 3D of an object often beforehand being selected (to move the book).

(iii) Rotation: rotation is the task of change of orientation of an object (to turn over the book).

2.2. Classification of the 3D Manipulation Techniques. There are several types of 3D manipulation techniques. These manipulation techniques can be structured by a classification, this classification can be used in order to better include/understand these techniques and also to evaluate them. There are classifications which have been proposed in order to structure the manipulation techniques, one can quote, for example, the classification by metaphor.

According to Fuchs an interaction metaphor is a symbolic system image of an action or a perception used to carry out a precise task in a VE. It is about the transposition of an object or a real concept in the virtual world [1]. One speaks, for example, about virtual hand metaphor: it is about the transposition of the hand of the user in the virtual environment. For Fuchs it is strongly advised to have recourse to a metaphor of interaction only when it is not possible to exploit a natural interaction directly [1].

(i) The egocentric metaphors: a metaphor egocentric is a metaphor in which the user acts directly from the interior of the virtual environment, as if it formed part of it. This type of metaphor is generally less appropriate to the manipulation tasks on a large scale, these metaphors are generally used for the manipulation of object with precision. The metaphors egocentric are divided into two families:

(a) virtual hand metaphors

(b) virtual pointer metaphors

(ii) The exocentric metaphors: they place the user on an external level. Hi interacts on the outside of the virtual environmet. Consequently these metaphors of interaction are particularly usable in the situations where the task is distributed at relatively large distances in the scene, such as the mobile objects. However, the manipulation of object which requires a very precise interaction, such as the deformation of object, will be more difficult with this type of metaphors.

2.3. User Performance in VE. Some user studies have been conducted in an attempt to investigate task performance when people use 3D interaction metaphors in single-user VEs.

Bowmann used testbed to evaluate $3 \mathrm{D}$ interaction metaphors like the ray casting, the GoGo, or the image plane, to carry out simple tasks and generic 3D interaction [4]. There are two tasks: (1) the selection, the user must select an object among a group of objects, the variables intrasubject variables for this task are the distance between the user and the targeted object ( 3 levels) and the size of object to be selected (2 levels). (2) Manipulation, the user must move and orient the object according to the information contained in a target. The intrasubject variables for this task are the relative size of the object compared to the final position (2 levels), and the degree of freedom, to test the effectiveness of the technique (2Dof, 6Dof). The results of this experiment show that the space skill and the former experiment in VE cannot be used to predict the performances of the users, the selection by occlusion can cause the tiredness of the arms, the male subjects had better performances, also the results show that manipulation by scaling can cause giddiness's after the experiment, the size and the distance do not significantly affect the user performance for pointing techniques.

Poupyrev also carried out an evaluation of the $3 \mathrm{D}$ interaction techniques [5]. In this experiment, the user must carry out two tasks: the selection and manipulation in order to evaluate two interaction metaphors: GoGo, and ray casting. The intrasubject variables for the selection are the distance between the user and the targeted object (5 levels), and the size of object to be selected ( 3 levels). The 
intrasubject variable for manipulation is the relative size of the object compared to the final position (3 levels). The results of this experiment show that the technique of ray casting gives better results than GoGo.

2.4. Empirical Studies in CVE. Various empirical studies have been done in CVE. The studies we quote are not exhaustive but they make it possible to depict some human factors which influence the interaction in a CVE. These factors are numerous.

The level of realism in CVE has been studied under different perspectives in order to investigate its impacts on social interaction in CVE. Gerhard showed that the degree of presence is higher when using an avatar of a humanoid type compared to an avatar of a shape type or a cartoon-type avatar [6]. Also, the use of a humanoid type generates more immersion, communication, engagement, and awareness. Nevertheless Garau showed that the use of a little anthropomorphic (attribution of human characteristics, or behavior to inanimate objects, animals, or natural phenomena) representation leads to a higher sense of copresence and social presence than the use of a precise anthropomorphism or no anthropomorphic representation [7]. Bailenson suggested that visual and behavioral realism must be carefully balanced [8].

In order to investigate small groups dynamics Slater conducted an experiment where groups of three people carried out a collaborative task [9]. Two participants used a simple screen (computer screen), and a third subject used a head-mounted display (HMD) device. The group performed a collaborative task in a face-to-face condition and in a virtual condition. The results of this study show that the participants with HMD developed leadership behavior. Casanueva study reports that the copresence score was much higher in the high-collaboration VE than in the lowcollaboration VE [10]. Also, Schroeder study shows that the immersed users naturally adopt dominant roles against users of desktop screens [11].

Rudlle in his studies $[12,13]$ looks at the verbal communication of participants in CVE. The authors used the problem of piano movers (maneuvering a large object through a restricted space). The task consisted of moving collaboratively an object from a starting point to an end point. Participants performed this task under two conditions: in a symmetric interaction where only the synchronized actions are allowed and in an asymmetric interaction and in two different configurations of the CVE, an offset CVE and a C-shaped CVE. The study reports that the subjects communicate better in the symmetric interaction condition than in the asymmetric one. It also reports that the speed and the direction of the movements of the hand coordination was poor under the two conditions.

In Hindmarsh et al. study [14] participants were asked to collaboratively arrange the layout of furniture in a virtual room and agree upon a single design. They were given conflicting priorities in order to encourage debate and discussion. This study shows that participants were able to make reference to objects in the shared environment through pointing gestures. However, problems of fragmentation were observed. These problems were due to a discontinuous visualization during the realization of the task caused by the desktop screen. In order to compensate for the fragmentation of the workspace, users increase their verbal communication using audio. Also Ruddle's et al. [12] study explains that a high quantity of verbal communication is employed to compensate for the fragmentation of the work place on a desktop screen.

Nakanishi compared the movement of users in three different conditions, that is, face-to-face, videoconference, and FreeWalk [15]. FreeWalk is a desktop meeting environment that provides a $3 \mathrm{D}$ community common where everybody can meet and can behave as in real life. Participants are represented as a pyramid of $3 \mathrm{D}$ polygons on which individual live video is mapped and can move freely. The results show that the participants have better communication in FreeWalk compared to the other two conditions. Participants also moved better in FreeWalk. Sallnas compared three types of communication (chat, audio, and audio-video) and their effects on presence. Their findings show that the level of social presence and virtual presence is higher with the audio condition. The audio-video users dialogue less than with chat and audio [16].

Most of the precedent studies use evaluation method for a single user in virtual environment. The impact of different avatar appearances on social interaction is among these studies. Most research works done studied design parameters in CVE research. Whereas for executing a specific task in CVE, it is necessary to have exchanges and interactions between the participants and the VE.

Understanding of these metaphors will help us to define the criteria, which are to be taken into account when designing a CVE. To our knowledge, a few studies have been conducted investigating the effectiveness of supporting teamwork between a geographically distributed group for the shard manipulation of objects $[17,18]$, the cooperative object manipulation [19]. But not the effect of 3D interaction metaphors on the user experience in a CVE-oriented task.

In collaborative scenario, a number of issues need to be addressed.

(i) How to maintain awareness of who is in the virtual environment, who the other users are, and what are they doing?

(ii) How to support nonverbal communication: pointing and gesturing?

The research question arising from these problems is the following:

"What is the impact of $3 D$ interaction metaphors on the user experience in a CVE-oriented task?"

To address this research question, we conducted an experimental study. In this study, we would systematically vary three 3D interaction metaphors (conditions or independent variables), (1) the virtual hand metaphor, (2) the ray casting metaphor, and (3) the GoGo metaphor, and investigate their impact on several dependent variables (collaborative effort, involvement, awareness, copresence, usability, satisfaction, and preference). 


\section{Experiment}

3.1. Task. In this experiment, participants will be grouped in pairs. We chose the collaborative task "3D puzzle," each pair will be asked to build collaboratively a 3D puzzle. The task requires the participants to work together in three conditions as a team to resolve the puzzle. The participants must build a 3D puzzle (of nine cubes) from an image, the goal of this puzzle is the color alignment of cubes. These cubes are initially positioned on a table in a random way and the participants can select these cubes and position them by using the $3 \mathrm{D}$ interaction metaphors on a skeleton in order to build the puzzle, see Figure 1.

This task has been chosen because it engages the participants to work together in order to find the good position of the cubes. That requires communication and a participation between the subjects. Also, this task makes it possible to use the interaction metaphors to select or manipulate the cubes.

This task is used in previous work and is considered a reference task in the studies of collaborative work. It is inspired by the famous task of Rubik's cube puzzle used in previous work. However, we have adapted this task to our research context below the main differences between our task and the task used in the Schroeder study. In the Schroeder study participants manipulate the cubes using avatars [11], then in our study we have chosen not to use avatars. We use 3D interaction metaphors.

Also the task used by Schroeder was to solve a puzzle involving 8 blocks with different colors on different sides and to rearrange the blocks such that each side would display a single color 4 squares of the same colour on each of the six sides [11], in our work participants must reconstruct an image by an assemblage of 9 cubes. The image to reconstruct corresponds to a gradient of colors.

During the experiment participants could not see their partners directly, but only the interaction metaphors represent the users in the virtual environment. They solely communicated by using the audio connection. We give the participants 15 minutes to solve the puzzle for each condition; if they exceed this time limit, we consider that they failed.

3.2. Independent Variables. In our study, we used three egocentric interaction metaphors. A metaphor of virtual pointer (ray casting) and two virtual hand metaphors type (a classic virtual hand metaphor and a GoGo metaphor). The choice of these metaphors is justified by the fact that they are the metaphors most representative of their family of metaphors, ray casting for the metaphors of virtual pointer and the virtual hand and GoGo for the virtual hand family of the metaphors. Also we decided to choose these three metaphors, they are used in the majority of the applications for the interaction 3D.

3.2.1. The Virtual Hand Metaphor. The virtual hand moves in the virtual environment based on the movement of the user's physical hand. A virtual object can be selected by touching or by intersection between the virtual hand and the object. The virtual object is then attached to the virtual hand and can then be manipulated. Manipulation of the object is done by direct transcription of the movements of the hand to the object see Figure 2.

3.2.2. The Ray Casting Metaphor. This metaphor allows the user to select an object by pointing to it with a virtual ray. The pointer direction was defined by the position and orientation of the user hand. Once selected, the virtual object can be manipulated as if it is attached to the ray see Figure 3.

3.2.3. The GoGo Metaphor. This metaphor gives the user an "elastic" virtual arm, to reach distant virtual objects. Space around the user is divided into two areas centered on him. When its hand evolves/moves in the proximal area, the "traditional" metaphor of virtual hand is used. When the user extends his hand beyond this zone, its movements are amplified and the coefficient of amplification increases when the arm of the user extends, see Figure 4.

3.3. Dependent Variables. According to our study, different dependent variables have been chosen. These variables are detailed here.

3.3.1. The Collaborative Effort. The collaborative effort is the work which two partners provide to achieve a specific task collaboratively. We use Biocca measure of collaborative effort [20]. Four statements addressed a perceived sense of collaborative effort, on a Likert scale from 1 to 7 . This questionnaire was used by Biocca in an experiment comparing face-to-face interaction with audio-video teleconferencing [20].

Questionnaire items:

(1) "My partner worked with me to complete the task,"

(2) "I did not help my partner very much?"

(3) "My partner did not help me very much,"

(4) "I worked with my partner to complete the task?"

3.3.2. Awareness/Involvement. In the case or two people being in the same VE, these people generate signs enabling them to have knowledge of the actions and intentions of their partner. This knowledge of the other which results from its interactions with the environment is often indicated in the literature by the "awareness." The awareness makes it possible for two partners to adapt and plan their behaviors according to what they mutually know of each other. According to Hofmann the involvement is a presence facets [14]. Involvement describes to what extent the participant's attentional resources are directed to the VE. We use Gerhard measure of awareness, four items captured the perceived sense of involvement and three items the awareness on a Likert scale from 1 to 7. This questionnaire was used by Gerhard to investigate the influence of the appearance of avatars on involvement and awareness. Subjects $(n=27)$ performed a collaborative judgment task [6]. 


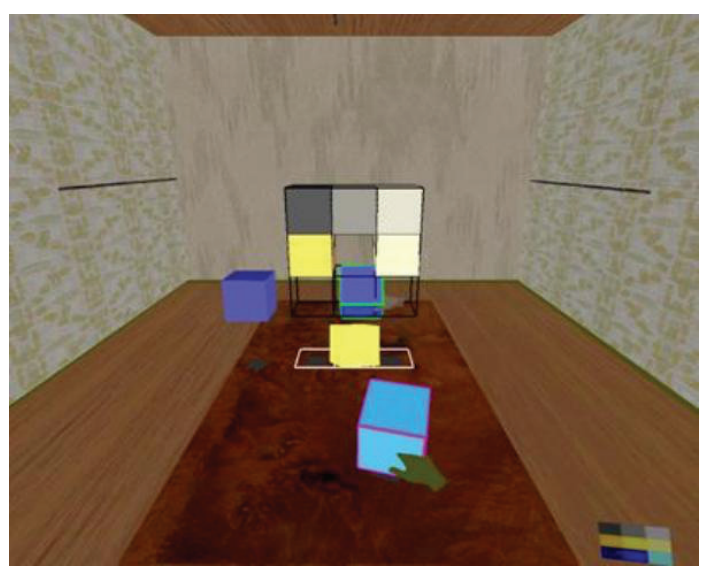

FIGURe 1: Virtual environment used.

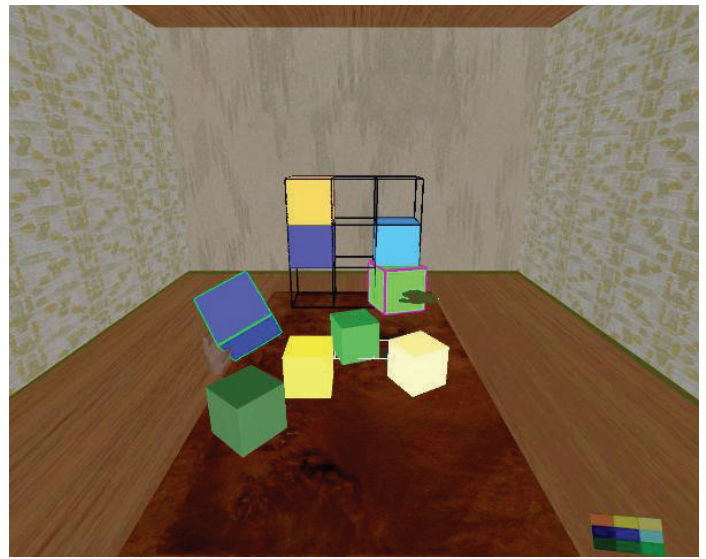

FIGURE 2: Screenshot of virtual hand metaphor.

Questionnaire items (involvement):

(1) "Were you involved in communication and the experimental task to the extent that you lost track of time?"

(2) "To what extent did events occurring outside the $3 D$ scene distract from your experience in the virtual environment?"

(3) "I was an active participant in the task,"

(4) "I enjoyed the virtual environment experience."

Questionnaire items (awareness):

(1) "I was aware of the actions of other participants,"

(2) "I was immediately aware of the existence of other participants,"

(3) "How aware were you of the existence of your virtual representation?"

3.3.3. Copresence. Copresence means the subjective sense of being together or being colocated with another person in a computer-generated environment. Two items address copresence, on a Likert scale from 1 to 7 , from the Schroder questionnaire [11].

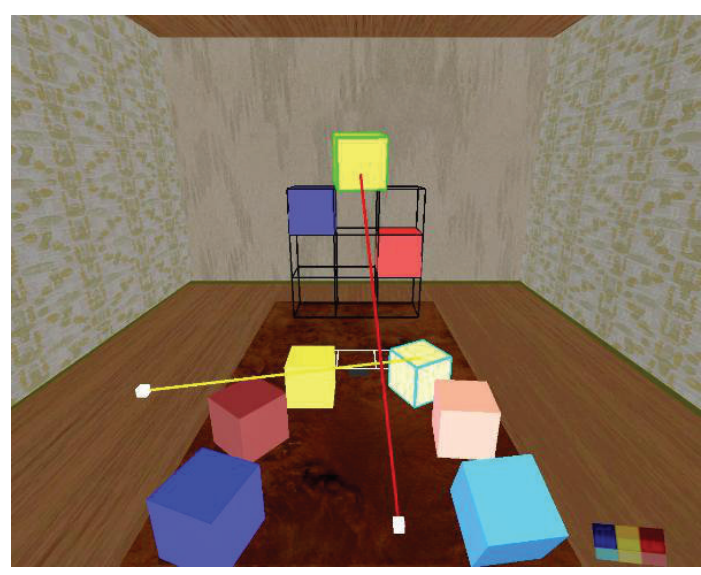

Figure 3: Screenshot of ray-casting metaphor.

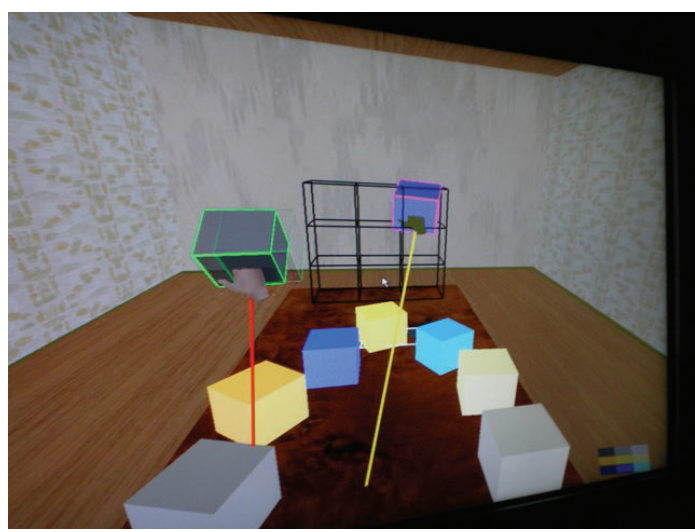

FIGURE 4: Screenshot of virtual GoGo metaphor.

Questionnaire items:

(1) "To what extent did you have a sense of being in the same room as your partner?"

(2) "When you continue to think back on the task, to what extent do you have a sense that you are together with your partner in the same room?"

3.3.4. Usability. According to Brooke, usability is a general quality of the appropriateness to a purpose of an artefact [21]. That means the context which a system is employed influences the usability of this system or tool [22]. Four items captured the usability of each metaphor, on a scale of 1 to 7 .

Questionnaire items:

(1) "It is easy to use this technique for selection/ manipulation?"

(2) "This interaction technique is flexible for selection/ manipulation?"

(3) "I can recover from mistakes quickly and easily?"

(4) "I used the interaction technique successfully every time?" 
3.3.5. Satisfaction. Three items addressed the satisfaction, on a Likert scale from 1 to 7.

Questionnaire items:

(1) "How satisfied are you by using this selection/ manipulation technique?"

(2) "I would recommend this interaction technique to a friend?"

(3) "This interaction technique is fun to use?"

3.3.6. Preference. User's preferences for the three conditions were assessed using four items. Questionnaire items:

(1) "If I had the choice when solving tasks like these I would choose:"

(2) "It was easiest for me to coordinate my actions with my partner when I used:"

(3) "It was easiest for me to predict my partner action when his/her used:"

(4) "It was easiest for me to manipulate objects when I used:"

3.4. Experimental Platform. For providing motion capture into our platform, we integrate a hybrid motion tracking system for full six degrees of freedom (6-DOF). This system is the result of the combination of our own $3 \mathrm{D}$ tracking system and the Nintendo Wiimote. Our 3D tracking system works with two infrared cameras and reflecting markers. Our tracking system uses stereoscopy where two cameras are used and equipped with infrared projector. The resulting monochromic images are processed in order to compute the $3 \mathrm{D}$ positions of the markers in realtime. The Nintendo Wiimote is a wireless versatile interaction device with several functions. We use this device for capturing orientation in the 3 axis.

Our collaborative platform is made of two Braccetto systems (see Figure 5). The platform is composed of a computer Intel Xeon CPU 3.0 GHz, equipped with two 1920 $\times 1080$ resolution LCD screens. The configuration of these two screens can be changed depending on the application.

These two systems are connected by UDP/IP network architecture (see Figure 6). The server is launched initially. It plays a double role. First of all, it must ensure the routing of the data of a transmitting client towards the other clients. Then, it must ensure of the safeguards of the VE state with regular time intervals.

3.5. Participants. We recruited the majority of our participants at the Macquarie University in Sydney, Australia. Participants were recruited via email advertisements and by flyers posted around the campus. The total number of participants was 32 (female participants represent $34.37 \%$ of the sample). Altogether, they were 16 sessions (two participants for each trial). The age of these participants was from 18 to 57 years with an average age (M) of 28.68 years and a standard deviation (SD) of 10.73. The necessary conditions for including potential participants were age more

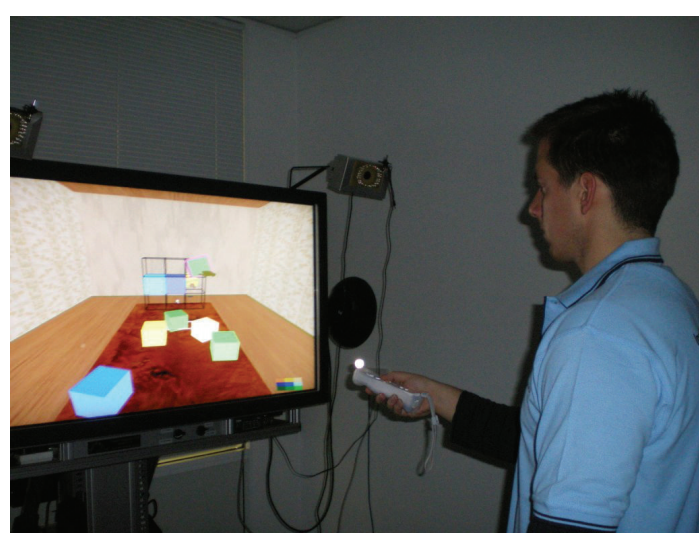

Figure 5: The experimental platform.

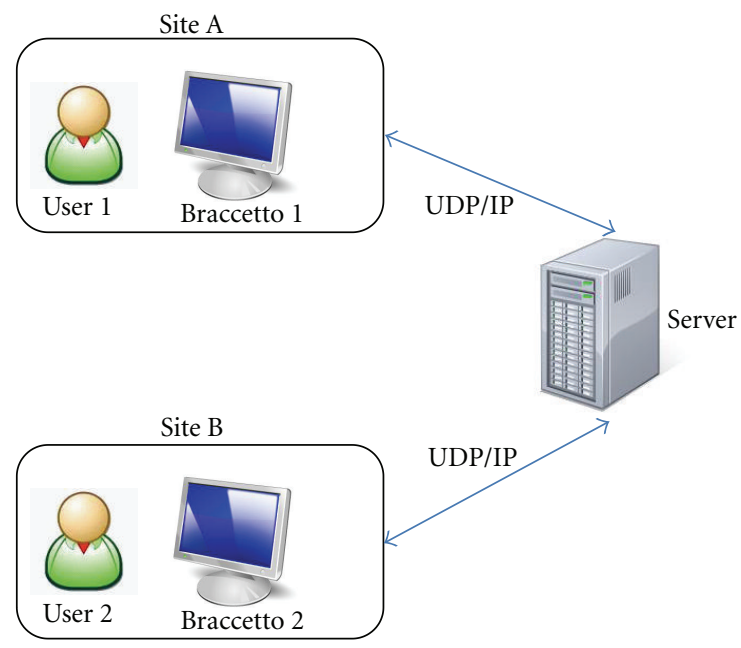

Figure 6: Diagram of the system architecture.

than 18 years, a normal or a correct vision, and fluent spoken English. At the end of the trial, each participant received a movie ticket for their participation.

3.6. Procedure. We placed the participants in two rooms that were approximately about thirty meters apart from one another. Before the experiment, participants read the general instructions. Then, they signed a consent form. Then we trained the participants in how to control the system and explained the use of each metaphor. This took approximately 10 to 15 minutes. They then answered an entry demographics questionnaire online to collect some details about the participants such as gender, age, occupation, proficiency of English language, video game experience, and previous use of Wiimote. We asked the participants in each session to carry out the task collaboratively by using interaction metaphors and to answer after each condition an online questionnaire. For the last condition they also answered an exit questionnaire to choose their preferences. Each trial lasted approximately 45 minutes. At the end of the experiment, the participants were brought together in 
one room for a debriefing and to ask them about their experiences and impressions of the trial.

3.7. Hypotheses. We conducted a pilot study with 4 trials, 4 weeks before the actual experiment, from the first impression of the users and the questionnaire results, we derived 3 general hypothese.

Hypothesis 1. "The ray casting metaphor increases copresence and awareness more than the virtual hand metaphor."

Hypothesis 2. "The ray casting metaphor leads to higher involvement level than that of the virtual hand."

Hypothesis 3. "The GoGo metaphor leads to higher collaborative effort level than that of the virtual hand and ray casting metaphors."

3.8. Results. The results presented in this section have been analyzed using SPSS version 16 . We used one-way ANOVA to compare mean differences using both 5\% and 1\% confidence levels. We used Scheffe post hoc comparisons to determine which pairs of groups are significantly different. Also, we used a Person's correlation analysis to investigate the relationship between dependent variables.

3.8.1. Copresence. The average copresence value of the participant using the ray casting metaphor is 4.90 , compared to 3.81 of the GoGo metaphor user and 3.75 for the virtual hand metaphor user. The differences between the three groups are significant $(F(2,93)=3.96, P=0.022$, see Figure 7). Post hoc testing revealed that ray casting and virtual hand are significantly different $(P=0.048)$ see Table $1(a)$.

3.8.2. Involvement. The average involvement value of the participant using the ray casting metaphor is 4.96 , compared 4.40 of the GoGo metaphor user and 4.15 for the virtual hand metaphor user. The differences between the three groups are significant $(F(2,93)=4.79, P=0.01$, see Figure 8$)$. Post hoc testing revealed that Ray casting and Virtual hand are significantly different $(P=0.013)$, see Table $1(b)$.

3.8.3. Awareness. The average awareness value of the participant using the ray casting metaphor is 5.73 , compared to 5.29 of the GoGo metaphor user and 4.90 for the virtual hand metaphor user. The differences between the three groups are significant $(F(2,93)=4.50, P=0.014$, see Figure 9). Post hoc testing revealed that Ray casting and virtual hand are significantly different $(P=0.014)$, see Table $1(\mathrm{c})$.

3.8.4. Collaborative Effort. The average collaborative effort value of the participant using the GoGo metaphor is 4.56, compared to 4.19 of the virtual hand metaphor user and 4.02 for the ray casting metaphor user. The differences between the three groups are significant $(F(2,93)=3.31, P=0.041$, see Figure 10). Post hoc testing revealed that ray casting and GoGo are significantly different $(P=0.046)$, see Table $1(\mathrm{~d})$.

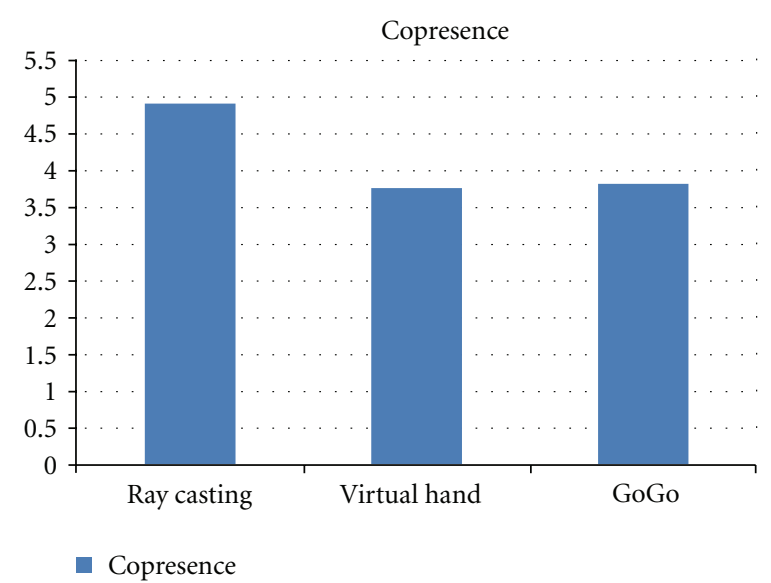

Figure 7: Mean difference in copresence.

Table 1: Post hoc Comparisons between conditions.

(a)

\begin{tabular}{lcccc}
\hline Factor & $F$ & df & $P$ & Post hoc comparisons \\
\hline Copresence & 3.96 & 2 & 0.022 & ray casting $>$ virtual hand \\
\hline
\end{tabular}

(b)

\begin{tabular}{lcccc}
\hline Factor & $F$ & df & $P$ & Post hoc comparisons \\
\hline Involvement & 4.79 & 2 & 0.01 & ray casting $>$ virtual hand \\
\hline
\end{tabular}

(c)

\begin{tabular}{lccccc}
\hline Factor & $F$ & df & $P$ & \multicolumn{2}{c}{ Post hoc comparisons } \\
\hline Awareness & 4.50 & 2 & 0.014 & ray casting $>$ virtual hand \\
\hline \multicolumn{5}{c}{ (d) } \\
\hline Factor & $F$ & df & $P$ & Post hoc comparisons \\
\hline Collaborative effort & 3.31 & 2 & 0.041 & GoGo $>$ ray casting \\
\hline
\end{tabular}

(e)

\begin{tabular}{lcccc}
\hline Factor & $F$ & df & $P$ & Post hoc comparisons \\
\hline Satisfaction & 3.74 & 2 & 0.027 & GoGo $>$ ray casting \\
\hline
\end{tabular}

(f)

\begin{tabular}{lcccc}
\hline Factor & $F$ & df & $P$ & Post hoc comparisons \\
\hline Usability & 3.18 & 2 & 0.046 & GoGo $>$ ray casting \\
\hline
\end{tabular}

3.8.5. Satisfaction. The average collaborative effort value of the participant using the GoGo metaphor is 4.76, compared 3.77 of the virtual hand metaphor user and 3.53 for the ray casting metaphor user. The differences between the three groups are significant $(F(2,93)=3.74, P=0.027$, see Figure 11). Post hoc testing revealed that ray casting and GoGo are significantly different $(P=0.04)$, see Table $1(\mathrm{e})$.

3.8.6. Usability. The average collaborative effort value of the participant using the GoGo metaphor is 4.36, compared to 3.87 of the virtual hand metaphor user and 3.34 for the ray casting metaphor user. The differences between the three 


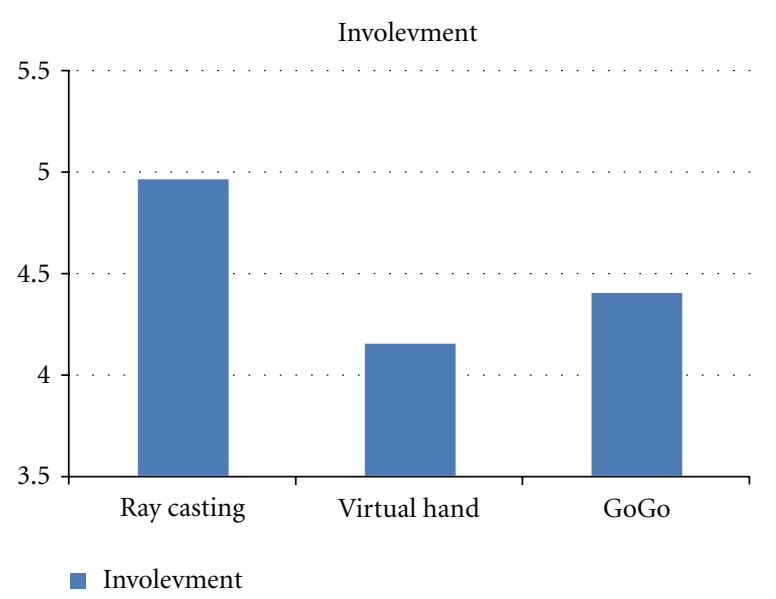

FIGURE 8: Mean difference in the involvement.

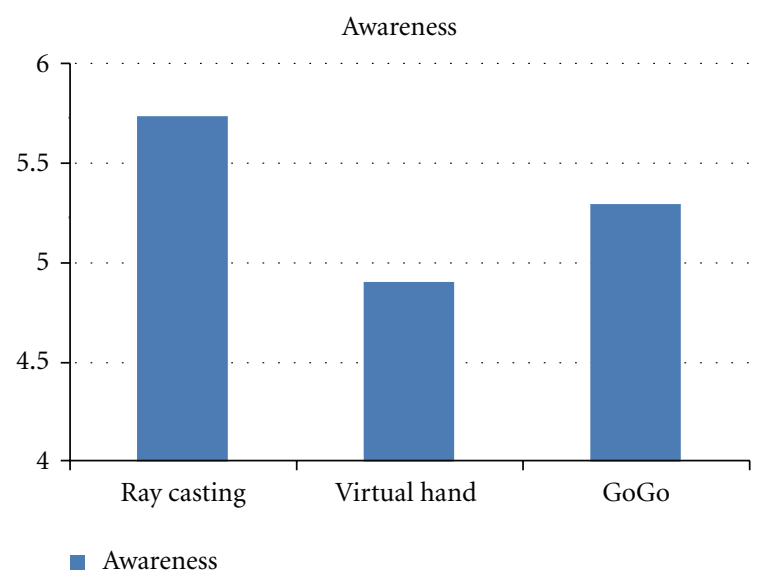

FIGURE 9: Mean difference in the awareness.

groups are significant $(F(2,93)=3.18, P=0.046$, see Figure 12). Post hoc testing revealed that ray casting and GoGo are significantly different $(P=0.046)$, see Table $1(f)$.

3.8.7. Preference. The analysis of the results of the questionnaire about relating to the user preference shows that $56.25 \%$ of users prefer the GoGo metaphor, $31.25 \%$ of the participants preferred the virtual hand metaphor and $12.5 \%$ of participants preferred the ray casting metaphor.

3.8.8. Correlation. A Person's correlation analysis was performed between the various variables, in each condition, to check if there were significant relationships between them. We obtained the following results ( see Table 2 ).

\section{Discussion}

The results of this study showed significant differences between the three conditions (virtual hand condition, ray casting condition, and GoGo condition) with respect to the dependent variables chosen (awareness, copresence, collaborative effort, satisfaction, usability, and involvement).

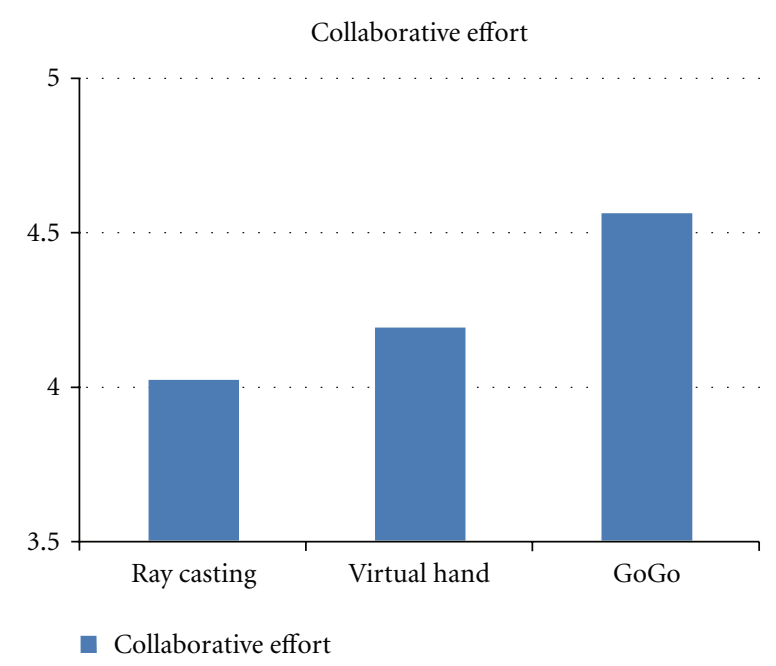

Figure 10: Mean difference in the collaborative effort.

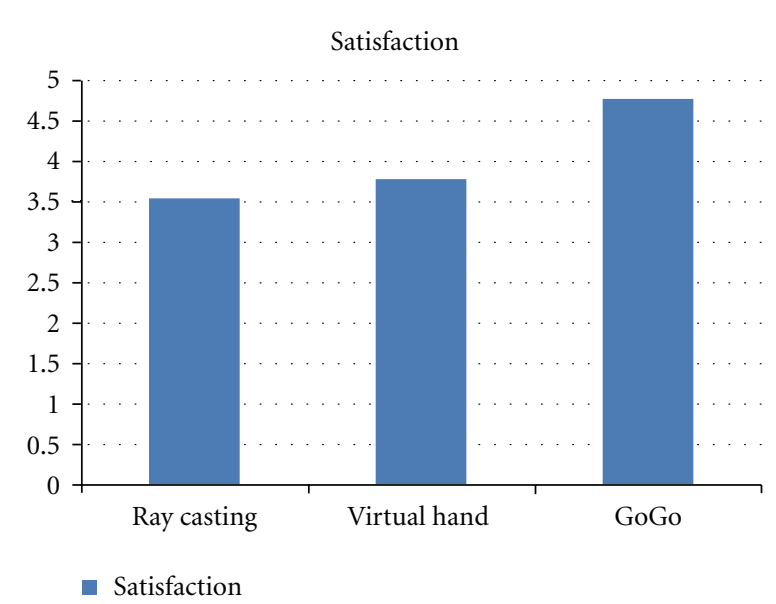

Figure 11: Mean difference in the satisfaction.

The statistical result of this experiment shows that the level of copresence is higher by using the ray casting metaphor compared to the GoGo and virtual hand metaphors, the post hoc test shows a significant difference between ray casting and the virtual hand. This result confirms Hypothesis 1. By analyzing the behavior of subject and during the debriefing meeting we noted a feeling of disappointment in using the virtual hand metaphor by the subjects, because they await a metaphor with more realistic behavior, for example, the fingers animation, closing or the opening of the hand, whereas the hand that used by us does not make it possible to have this type of behavior, it is a simple hand without animation (in our study we used a simple hand without animation). Nowak and Biocca study [23] shows that a low level of anthropomorphism gives a more important level of copresence and social presence than a higher level of anthropomorphism or null anthropomorphism. In fact, a high level of anthropomorphism induces more hopes which are usually not reached and implies a reduction of the presence. 
TABLE 2: Person's correlations between variables (legend: + = (positively) significant on 1\% confidence level).

\begin{tabular}{|c|c|c|c|c|}
\hline & Copresence & Involvement & Satisfaction & Usability \\
\hline \multirow{3}{*}{ Involvement } & + & & & \\
\hline & $r=0.512$ & & & \\
\hline & $P=0.001$ & & & \\
\hline \multirow{3}{*}{ Awareness } & + & + & & \\
\hline & $r=0.479$ & $r=0.599$ & & \\
\hline & $P=0.001$ & $P=0.001$ & & \\
\hline \multirow{3}{*}{ Satisfaction } & & + & & \\
\hline & & $r=0.406$ & & \\
\hline & & $P=0.001$ & & \\
\hline \multirow{3}{*}{ Usability } & & + & + & \\
\hline & & $r=0.309$ & $r=0.309$ & \\
\hline & & $P=0.001$ & $P=0.001$ & \\
\hline \multirow{3}{*}{ Collaborative effort } & & & + & + \\
\hline & & & $r=0.343$ & $r=0.264$ \\
\hline & & & $P=0.001$ & $P=0.001$ \\
\hline
\end{tabular}

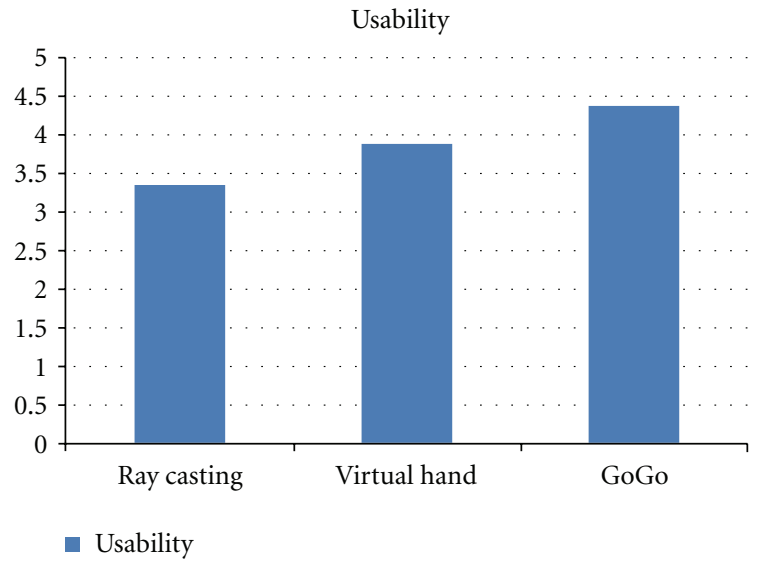

FIgURE 12: Mean difference in the usability.

The level of awareness is higher by using the ray casting metaphor (ray casting $>$ GoGo > virtual hand). The post hoc test shows a significant difference between ray casting and the virtual hand, during the realization of the task the metaphor of ray casting makes it possible to better include/understand the actions of the partner, this comprehension facilitates actions of the other taking part and facilitates the process of awareness. That justifies the fact that the level of awareness is higher by using ray casting compared to the virtual hand. Also we found a significant correlation between copresence and awareness. According to Ruth [24], copresence refers to the mutual awareness between participants. This explains the relationship between the copresence and the awareness.

The ray casting metaphor gives a level of involvement higher than the other metaphors (ray casting > GoGo > virtual hand). this confirms Hypothesis 2. The analysis of the results of post hoc raises a difference significant between the ray casting metaphor and the virtual hand metaphor. Also the results show a positive correlation between involvement, and the copresence but also between involvement and the awareness. The copresence, the involvement and the awareness are three concepts which are dependent. This result is logical, indeed these three concepts take part in the psychological acceptance process of the user presence in a CVE.

We noticed that there is a positively significant relationship between usability and satisfaction, usability and satisfaction are higher when using the GoGo condition, compared to the virtual hand and the ray casting conditions. At the debriefing time the majority of the participants announced that the GoGo metaphor is an intuitive metaphor pleasant to use compared to the other metaphors. Indeed the virtual hand metaphor represents the advantage of being simple and natural to use, but the disadvantage raises of this metaphor does not make it possible to select the objects which are far from the user. The ray casting metaphor arises well for the selection of the objects moved away, but rotation is difficult with this metaphor whereas the GoGo metaphor is a metaphor which makes it possible to select and to handle objects which are far from the user in a simple and intuitive way. That justifies the fact of it having a higher level of satisfaction and usability (GoGo $>$ hand virtual $>$ ray casting).

The perception of the collaborative effort is higher with the GoGo metaphor (GoGo > hand virtual > ray casting), this result confirms Hypothesis 3; as we mentioned previously this metaphor represents the advantage of being intuitive and pleasant to use, that creates in the participants a feeling of motivation to achieve the task, this motivation results in the collaboration effort which increases by using the GoGo metaphor.

We also find that the collaborative effort and satisfaction and the collaborative effort and the usability are positively correlated. When an interaction metaphor has the advantage 
of being easy to use, and the user is satisfied by the use of the metaphor, that motivates the participants and supports the process of collaboration.

The work completed in this experimental study enabled us to show the importance of the choice of the interaction metaphor. Also thanks to the results of our experiment, we could draw up a list of recommendation to support the $3 \mathrm{D}$ interaction in a CVE where the human factor elements are the essential elements in the loop of the design of CVEs.

(i) A direct control of the user actions, to have an interaction metaphor with a direct control makes it more interactive and supports the collaboration in the CVE.

(ii) Use interaction metaphors with a high level of cognitive effort only when necessary. An interaction metaphor with a high cognitive load requires of the user to make considerable mental effort, this effort decreases the collaboration level between the participants for the achievement of the task.

(iii) To have a balance between visual and behavioral realism. We note that if behavioral realism in adequacy with visual realism is not used in the interaction metaphor, it creates in the participants a feeling of disappointment, which influences in a negative way the copresence, the involvement, and the awareness.

(iv) It is important to take into account the ergonomic factors of the interaction metaphor. These factors influence the usability, satisfaction, and the collaborative effort. It is thus necessary to use interaction metaphors that are intuitive and pleasant to use.

We chose three interaction metaphors according to criteria which correspond to the context of our application (the type of the input device and the output display), we think that it would be interesting to evaluate the interaction metaphors in other types of environment like the CAVE or the head-mounted display HMD.

In this work, we did not use any peripherals with haptic feedback, it would be interesting to be interested in the collaborative interaction, by using these peripherals in an EVC on scale 1, like the Spidar [25].

Finally the number of users in our experiments was limited to two users whereas situations of collaborative work ask for the intervention of more than two users, it would be interesting to have a collaborative platforms with more than two users, in order to study various problems like the management of the turn taking, the management of the conflicts, and the intentions of the participants.

\section{Conclusion}

This paper reports the results of an experiment study conducted to evaluate the impact of 3D interaction metaphors on the user experience in a CVE-oriented task. The results demonstrate that there is a significant difference by changing the interaction metaphor on the copresence, awareness, involvement, collaborative effort, satisfaction usability, and preference. The results confirm our overall working hypothesis, that the choice of $3 \mathrm{D}$ interaction metaphor affects significantly user's experience in CVE.

In that way, we hope that such research works will help to improve the design of a new enjoyable and efficient CVE generation.

\section{References}

[1] S. Coquillart, B. Arnaldi, A. Berthoz et al., Le Trait de la Ralit Virtuelle: Interfaage, Immersion et Interaction en Environnement Virtuel, vol. 2, Mines de Paris, 2006.

[2] R. M. Mark, "ISAAC: a virtual environment tool for the interactive construction of virtual worlds," Technical Report, University of North Carolina, Chapel Hill, NC, USA, 1995.

[3] D. A. Bowman, E. Kruijff, J. J. Laviola, and I. Poupyrev, 3D User Interfaces: Theory and Practice, Addison-Wesley Educational Publishers, 2004.

[4] D. A. Bowman, D. B. Johnson, and L. F. Hodges, "Testbed evaluation of virtual environment interaction techniques," in Proceedings of the ACM Symposium on Virtual Reality Software and Technology (VRST '99), pp. 26-33, ACM, New York, NY, USA, December 1999.

[5] I. Poupyrev, S. Weghorst, M. Billinghurst, and T. Ichikawa, "A framework and testbed for studying manipulation techniques for immersive VR," in Proceedings of the ACM Symposium on Virtual Reality Software and Technology (VRST'97), pp. 21-28, September 1997.

[6] M. Gerhard, D. J. Moore, and D. J. Hobbs, "Continuous presence in collaborative virtual environments: towards a hybrid avatar-agent model for user representation," in Proceedings of the 3rd International Workshop on Intelligent Virtual Agents, pp. 137-155, 2001.

[7] M. Garau, M. Slater, V. Vinayagamoorthy, A. Brogni, A. Steed, and M. A. Sasse, "The impact of avatar realism and eye gaze control on perceived quality of communication in a shared immersive virtual environment," in Proceedings of the SIGCHI conference on Human factors in computing systems (CHI '03), pp. 529-536, ACM, New York, NY, USA, April 2003.

[8] J. N. Bailenson, K. Swinth, C. Hoyt, S. Persky, A. Dimov, and J. Blascovich, "The independent and interactive effects of embodied-agent appearance and behavior on self-report, cognitive, and behavioral markers of copresence in immersive virtual environments," Presence: Teleoperators and Virtual Environments, vol. 14, no. 4, pp. 379-393, 2005.

[9] M. Slater, A. Sadagic, M. Usoh, and R. Schroeder, "Smallgroup behavior in a virtual and real environment: a comparative study," Presence: Teleoperators and Virtual Environments, vol. 9, no. 1, pp. 37-51, 2000.

[10] J. S. Casanueva and E. H. Blake, "Small group experiments in collaborative virtual environments," 2000.

[11] R. Schroeder, A. Steed, A. S. Axelsson et al., "Collaborating in networked immersive spaces: as good as being there together?" Computers and Graphics, vol. 25, no. 5, pp. 781-788, 2001.

[12] R. A. Ruddle, J. C. Savage, and D. M. Jones, "Symmetric and asymmetric action integration during cooperative object manipulation in virtual environments," ACM Transactions on Computer-Human Interaction, vol. 9, pp. 285-308, 2002.

[13] R. A. Ruddle, J. C. D. Savage, and D. M. Jones, "Verbal communication during cooperative object manipulation," in Proceedings of the ACM Conference on Collaborative Virtual Environments (CVE'02), pp. 120-127, October 2002. 
[14] J. Hindmarsh, M. Fraser, C. Heath, S. Benford, and C. Greenhalgh, "Object focused interaction in collaborative virtual environments," ACM Transactions on Computer-Human Interaction, vol. 7, pp. 477-509, 2000.

[15] H. Nakanishi, C. Yoshida, T. Nishimura, and T. Ishida, FreeWalk: A Three-Dimensional Meeting-Place for Communities, 1998.

[16] E. L. Sallnäs, "Effects of communication mode on social presence, virtual presence, and performance in collaborative virtual environments," Presence: Teleoperators and Virtual Environments, vol. 14, no. 4, pp. 434-449, 2005.

[17] D. Roberts, R. Wolff, O. Otto, and A. Steed, "Constructing a gazebo: supporting teamwork in a tightly coupled, distributed task in virtual reality," Presence: Teleoperators and Virtual Environments, vol. 12, no. 6, pp. 644-657, 2003.

[18] R. Wolff, D. J. Roberts, and O. Otto, "A study of event traffic during the shared manipulation of objects within a collaborative virtual environment," Presence: Teleoperators and Virtual Environments, vol. 13, no. 3, pp. 251-262, 2004.

[19] M. S. Pinho, D. A. Bowman, and C. M. D. S. Freitas, "Cooperative object manipulation in immersive virtual environments: framework and techniques," in Proceedings of the ACM Symposium on Virtual Reality Software and Technology (VRST '02), pp. 171-178, ACM, New York, NY, USA, November 2002.

[20] F. Biocca, C. Harms, and J. Gregg, "The networked minds measure of social presence: pilot test of the factor structure and concurrent validity," in Proceedings of the 4th Annual International Workshop, pp. 9-11, Presence, Philadelphia, Pa, USA, May 2001.

[21] J. Brooke, System Usability Scale.

[22] E. van Wyk and R. de Villiers, "Usability context analysis for virtual reality training in South African mines," in Proceedings of the Annual Research Conference of the South African Institute of Computer Scientists and Information Technologists on IT Research in Developing Countries (SAICSIT '08), vol. 338, pp. 276-285, ACM, New York, NY, USA, 2008.

[23] F. Nowak and K. L. Biocca, "The effect of the agency and thropomorphism of users sense of telepresence, copresece, and social presence in virtual environment," Presence: Teleopers and Virtual Environments, vol. 12, no. 5, pp. 481-494, 2003.

[24] R. Ruth, "Social presence as presentation of self," in Proceedings of the 8th Annual International Workshop, pp. 21-23, Presence, 2005.

[25] L. Buoguila, M. Ishii, and M. Sato, "Multi-modal haptic device for large-scale virtual environment," in Proceedings of the 8th ACM International Conference on Multimedia (ACM Multimedia 2000), pp. 277-283, USA, November 2000. 

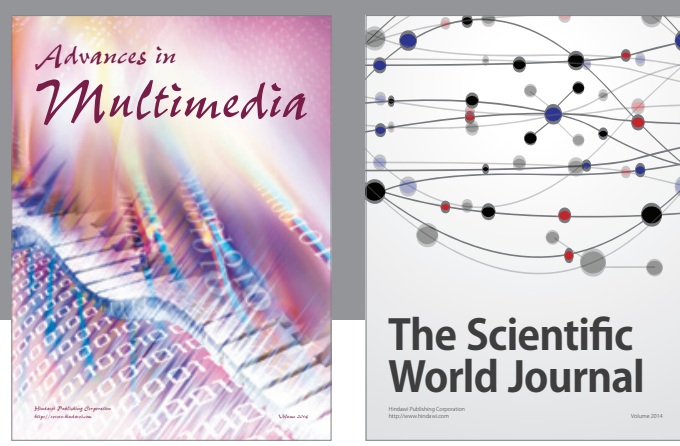

The Scientific World Journal
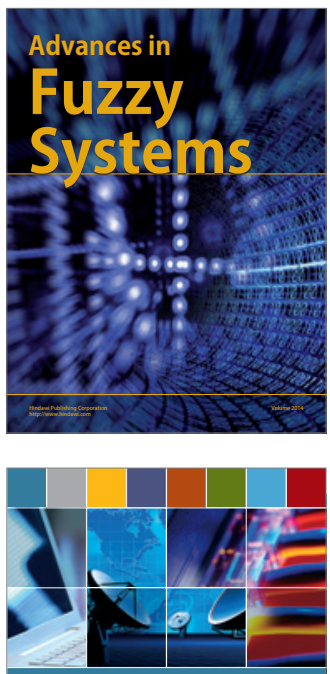

Computer Networks and Communications
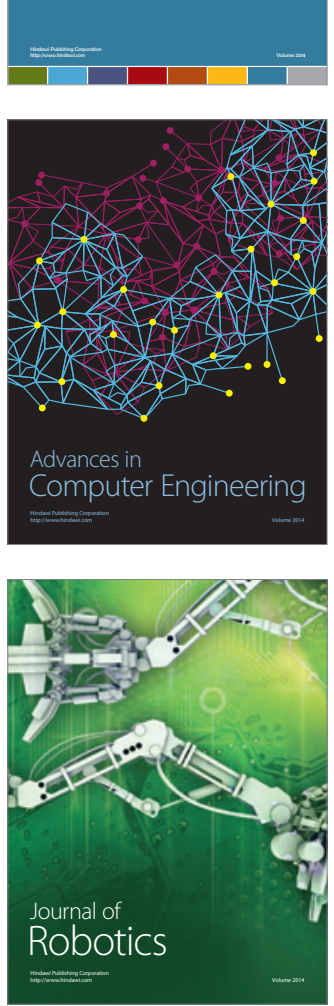
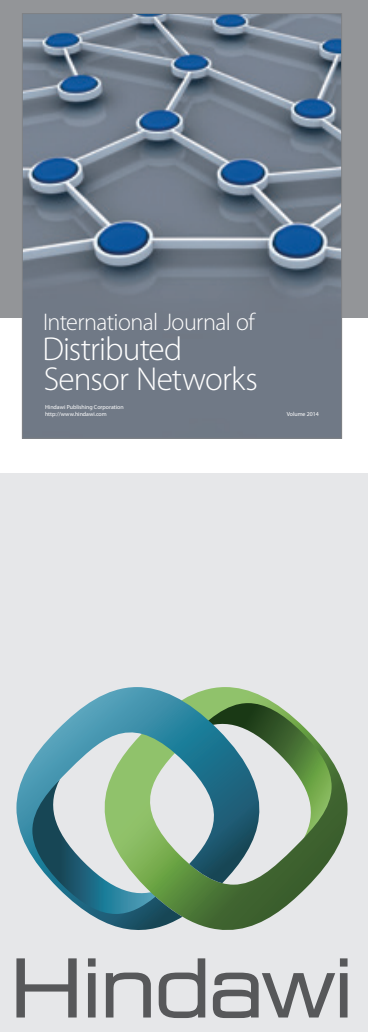

Submit your manuscripts at

http://www.hindawi.com
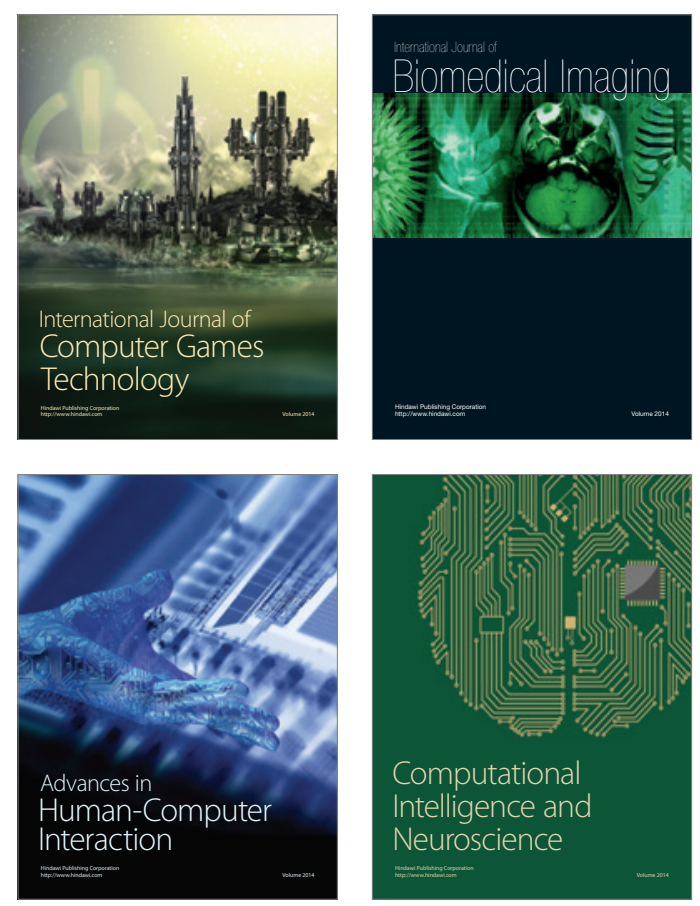
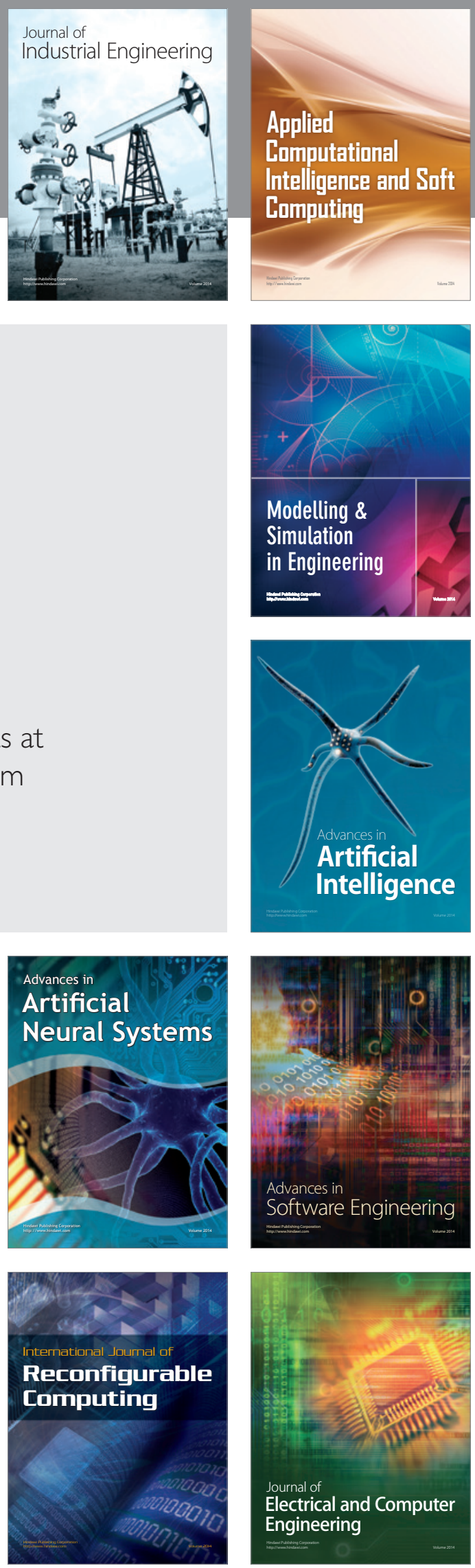